\title{
Differential Spatio-temporal Multiband Satellite Image Clustering using K-means Optimization With Reinforcement Programming
}

\section{Irene Erlyn Wina Rachmawan, Ali Ridho Barakbah, Tri Harsono}

\author{
Postgraduate Applied Engineering of Technology \\ Division of Information and Computer Engineering, Department of Information and \\ Computer Engineering, Electronic Engineering Polytechnic Institute of Surabaya \\ EEPIS Campus, Jalan Raya ITS, Sukolilo 60111, Indonesia \\ E-mail: ireneerlyn@pasca.student.pens.ac.id, \{ridho, trison\}@eepis-its.edu
}

\begin{abstract}
Deforestration is one of the crucial issues in Indonesia because now Indonesia has world's highest deforestation rate. In other hand, multispectral image delivers a great source of data for studying spatial and temporal changeability of the environmental such as deforestration area. This research present differential image processing methods for detecting nature change of deforestration. Our differential image processing algorithms extract and indicating area automatically. The feature of our proposed idea produce extracted information from multiband satellite image and calculate the area of deforestration by years with calculating data using temporal dataset. Yet, multiband satellite image consists of big data size that were difficult to be handled for segmentation. Commonly, KMeans clustering is considered to be a powerfull clustering algorithm because of its ability to clustering big data. However K-Means has sensitivity of its first generated centroids, which could lead into a bad performance. In this paper we propose a new approach to optimize K-Means clustering using Reinforcement Programming in order to clustering multispectral image. We build a new mechanism for generating initial centroids by implementing exploration and exploitation knowledge from Reinforcement Programming. This optimization will lead a better result for K-means data cluster. We select multispectral image from Landsat 7 in past ten years in Medawai, Borneo, Indonesia, and apply two segmentation areas consist of deforestration land and forest field. We made series of experiments and compared the experimental results of K-means using Reinforcement Programming as optimizing initiate centroid and normal K-means without optimization process.
\end{abstract}

Keywords: Deforestration, Multispectral images, landsat, automatic clustering, K-means. 


\section{INTRODUCTION}

Multispectral image delivers great source of data for studying temporal changeability of earth environment. It is used in a number of implementation such as environmental damage, nursing of land use, radiation level check, urban planning, growth directive, soil test and crop outcome increment [1]. One major area of multispectral image analysis is vegetation mapping classification. This area delivering information about forestation, deforestation or the changes of vegetation on earth. Multispectral image provide an information which could delivers a good coverage, mapping and classification about land cover features like vegetation, soil, water and forest information. Nowadays, Multispectral image analysis became a trend and replace the manual classification techniques for Mapping area in earth surface, which the manual classification techniques necessitates expensive and time-intensive field surveys [8]. Because of this trend, many researches and studies has been done on working in multispectral image classification. Classification result will shows identifiable or meaningful features of land cover area [9]. The result could be implied in a bunch of information kind, depends on how the information will serve. Regardless of all the advantages, Multispectral image classification is a difficult things to do, because of the complexity of landscapes captured and the large data size of images.

Multispectral images consist of multiband Landsat satellite image, it is composed from seven bands or layers, each band represent a different portion of electromagnetic spectrum. This seven bands will be used as one dataset before processing in segmentation algorithm. Multispectral image classification project done by combining image processing and unsupervised classification methods or clustering. Multispectral classification algorithms have gained attention in the recent days, due to their good performance in showing desire information from Landsat images. Multispectral clustering result can be receive by grouping area in multispectral images by comparing and grouping the similarities between each area. Multispectral clustering will produce similar object and generate a new image of clustered image. Cluster algorithm will specify and identify new cluster of items with a high degree of similarity toward each item (internal homogeneity) and a not likely members of other clusters (external homogeneity).

However, Landsat images have a big data size and has colour feature that tend to be similar with the other bands, that were difficult to handle In other hand, K-Means discovered as one of most well-known methods for clustering, it was developed by Mac Queen in 1967. The simplicity of Kmeans made this algorithm could be implemented in various cases that has a big size data. K-means is a partition clustering method that separates data into k groups. This reason makes k-means method became very popular to be implemented because of its ability to cluster huge and large data size with quickly and efficiently. However, K-means algorithm still has lack in its flow, $\mathrm{K}$-means very sensitive in first-generated centroid. Original K-means algorithm generate its first centroid randomly, when the value of random 
initial centroid has close distance with correct data, K-means will have high possibility to find out the optimal centroid. Otherwise, if the initial centroid was random incorrect value, K-Means will lead to incorrect clustering group results [11]. Because of initial starting points generated randomly, K-means does not always guarantee the optimal clustering result [12]. In some case Kmeans method will not reach global optimum, but only in local optimum value.

\section{RELATED WORKS}

Several methods proposed cluster initialization for K-means to solve multispectral images. Modification of K-means, Coting K-means is discussed by James and Galen [10] by expressed as a sum of discontiguities at each pixel for easy compute, using fixed k cluster. Remote sensing offers the possibility to analyze changes in spatial structure at the scale of landscapes. Indicators of the degradation of the vegetation cover can be derived from such measures. For example, Pickup \& Foran [12] developed a method to monitor and landscapes used for pastoralism based on the spatial variability of the vegetation. The spatial autocorrelation function and mean-variance plots of a spectral indicator were found to be successful in discriminating between the cover responses typical of good and poor rainfall years. For drought conditions, the decrease in spatial autocorrelation with increasing spatial lag was rapid since the ground surface is bare and most of the vegetation signal comes from scattered areas of trees and shrubs. A low decay rate of the autocorrelation function indicated a greater spatial uniformity of the landscape, e.g. during wet periods, when more ground cover is present so reducing the contrast between the bare soil signal and that produced by trees and shrubs. Similar observations were made by Lambin [12] over the seasonal and inter-annual cycle of three West African landscapes. Vogt also analyzed the seasonal changes in spatial structure of a West African landscape, showing that there is a marked seasonal cycle in the spatial structure of a vegetation index (NDVI), surface temperature and albedo, and that zones of ecological transition have an identifiable seasonal dynamic in spatial structure. However, the monitoring of these spatial variability measures only provides for a qualitative description of the cover state. Lambin \& Strahler [12] showed that the detection by remote sensing of land-cover change processes is Index and suface temp) and spatial indicators of surface condition. Their study suggested that the detection of inter-annual changes in landscape spatial structure is more likely to reveal long term and long lasting land-cover changes while spectral indicators are more sensitive to fluctuations in primary productivity associated with the inter-annual variability in climatic conditions. The long term monitoring of landscape spatial pattern in addition to other biophysical variables can thus lead to the detection of a greater range of processes of landscape modification. Some optimization algorithm also has been observed by many scientific. One of the researcher implement centroid optimization by combining kmeans and 
hierarical clustering, despite the good performaces and faster speed toward another algorithm, the experiment shows its solution could be trapted in local optima [11]. In this paper, we propose a new approach, combining Reinforcement Programming to optimizing K-means first initial centroid.

\section{ORIGINALITY}

Deforestration is a critical issues in Indonesia, it could be identified by Multispectral images. Multispectral images is a source of data for indicating the temporal changeability of earth environment. Many research for segementing multispectral images have been done by using classification and clustering methods. K-means as clustering methods have fast computational time for clustering a high number of data, but need to adjust and optimize first initiate centroids to produce high precission.

In this paper, we propose a new approach for clustering multispectral images using K-means which can perform clustering in fast computational time with high accuracy in detecting deforestration area. We use Reinforcement Programming to optimize K-means first initial centroid.

Reinforcement Programming is expected to optimize initiate centroid to set high accuracy of cluster result using K-means. As for result, clustered temporal dataset will be calculated by simple differential process to indicate the change of deforestration area in temporal time. And calculate the deforestration area by counting pixels of clustered area which has deforestration area as a label and multiply by satellite scale.

\section{SYSTEM DESIGN}

The proposed system consist of 3 phases: (1) Data pre-processing phase, (2) RP algorithm to generate initial centroid, (3) Kmeans and (4) differentiate process to measure deforestration area in temporal time. The general Diagram System shown in Figure 1.

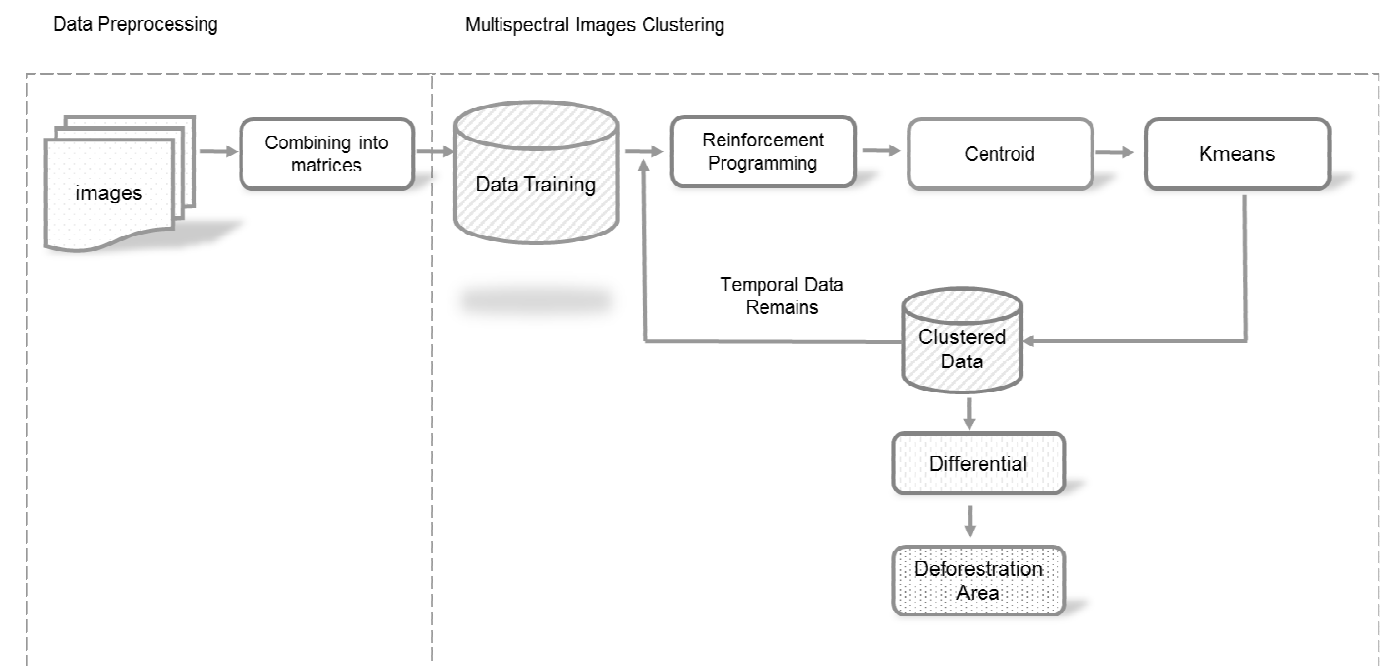

Figure 1. The general Diagram System 


\subsection{Data Pre-processing Phase}

The proposed system using real satellite image data from landsat satellite. With total dataset consist of 6 band where each band contains 2000 x 2300 pixels. As for preparing the data training, combining process will take color per pixel in each band and listed into a new dataset. So, at the end of data pre-processing, a clean matriks where contains data for each band is ready for clustering process.

\subsection{RP Algorithm to Generate Initiate Centroid}

In this phase, a new approach to generating initial centroids for $\mathrm{K}$ means is stated, by using Reinforcement Programming, as a new approach for solving K-means by modifying Reinforcement Learning to solve a function based problem[12]. Reinforcement Programming (RP) algorithm is the algorithm using basic concept of reinforcement learning. So in its implementation, Reinforcement programming has the same behaviour of Reinforcement Learning, by involving a balance between exploration of uncharted territory and exploitation of current knowledge to find solution. The solution determined by as much reward as possible in process learning. Reward and punishment are a value given by environment from the agent step.

In RP, this policy is learned through trial-and-error interactions of the agent with its environment: on each interaction step the agent senses the current states of the environment, chooses an action to perform, executes this action, altering the states of the environment, and receives a scalar reinforcement signal $r$ (a reward or penalty). The benefits of Reinforcement Programming is to bring a benefit in optimization case using an intelligent learning approach based on Reinforcement Learning. With involving the characteristics of Reinforcement Learning, Reinforcement Programming provides an experience-based learning to achieve the global optimum.

\subsubsection{Main Variables of Reinforcement Programming}

Reinforcement Programming is mainly based on the Reinforcement Learning. A number of slight modifications of Reinforcement Programming can be formulated where:

- $\quad \beta$ : is a variable value to give impact to step that the agent will take.

- $\quad \mu$ : a variable that will set a step value to the last step.

The goal of the agent in a RP problem is to learn an optimal solution by set action $\leftarrow d_{p}$. step $p$ that action is accumulated from previous step with direction of step that will be taken. The current States will be assign in variable new $S \leftarrow$.New states will be accumulated by cuttent new states and action new $S_{p} \leftarrow$ new $S_{p}+$ action $\left(\right.$ new $S_{p}$ must be in [minval. maxval $]$ ]). And assign variable step with rule : 
new $S_{p} \leftarrow S_{p}+$ action

The RL technique is well-known uses a strategy to learn an optimal via learning of the action values. It iteratively approximates new States. In RP the condition of using exploitation or exploration are decided by random

$\operatorname{step}_{p} \leftarrow \operatorname{step}_{p}-\mu \cdot r_{p}$. step $_{p}$

$\mathrm{p}$ are probability to take action whether to exploit or explore a finite state. To balance exploitation and exploration $\mathrm{p}$ can be set in 0.5 . Variable on reinforcement Programming structure is given in Figure 2.

State is the first position of agent to start solving problem. State can be initiate as assigned value or random number, depends of cases that will handled by agent. Variable reward is an array to save reward of each state that has been declared. To change the action direction of agent we need to declare direction variable. In first position direction can be assign with positive number to increase the direction in positive grid line. Action value is an array to save the calculation of action that has been taken by agent. Variable above are formulating to have compatible with optimization cases. Modification of different heuristic case will change the condition of variable state and direction.

\subsubsection{System Architecture}

System architecture given in Figure 2, explain the flow of Reinforcement Programming while find the best solution for optimizing initiate centroid in K-means. The basic Reinforcement Programming algorithm starts with an initialization phase, where

i. Assign data item and set into variable dataset

ii. Set modelling state value calculation(depend on optimization cases)

iii. Set probability for exploration rate. Use 0.5 to get a balanced action for exploration and exploitation.

iv. Assign the value of new state in state.

v. The agents process state evaluation to whether receive reward or punishment for current action.

This is done using an index that stores the positions of all 'free' data items on the grid. The process is as shown in Figure 2. 


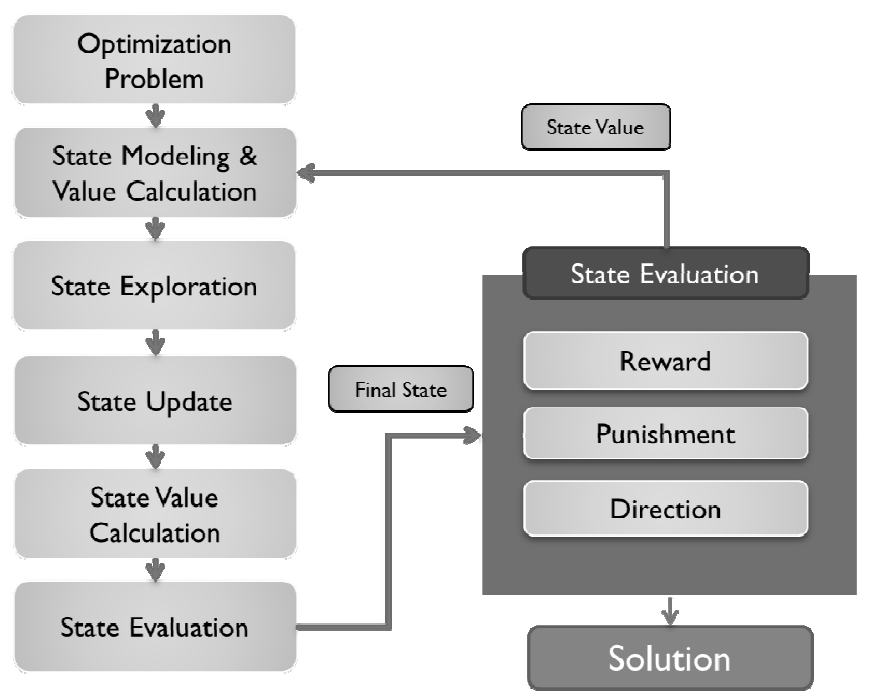

Figure 2. Step of Reinforcement Programming

First step, RP processing case based function by modelling state and state value calculation. Here, RP will randomly generate first initiated Centroid as state. As for state value calculation counted from variance of cluster homogienity. Then RP will begin state exploration in finite environment of problem, the actions that can be taken by agent is determined by exploration rate. If a random number is bigger than exploration rate than the agent will choose solution randomly in finite area. But if the random numbers are smaller than exploration rate than agent will consider taking an action based on reward. After take an action agent updates the current state and calculates current state value. A value from current state will label as reward or punishment, and determining the next direction of exploration. If agent receives punishment, then the agent will change its direction for the next exploration step. This process will be iterates in some value that already assign as number of learning time for agent of RP.

An extension of this algorithm is presented where the parameter is an adaptively updated during the execution of the algorithm. This algorithm is given in Figure 4. Reinforcement Learning needs to initialize dataset to identify problem environment. Agent will place randomly or assigned value depends on handled cases. After calculate state value, agent will determine action between exploration and exploitation. Then execute action and calculating new state value to determine reward based on state current value.

Reward of new state in the environment is computed through the following Equation (3) and (4)

$r_{p} \leftarrow r_{p}+\beta \cdot\left(1-r_{p}\right)$

The equation above show the formula for increasing reward value. 
$r_{p} \leftarrow r_{p}-\beta \cdot\left(1-r_{p}\right)$

And in case to give punishment, Reinforcement Programming used formula above to decrease reward value. Where $\beta$ is a constant to set a value of reward with scalar 0.1 until 1 . The more scalar that will be used it will impact the increase or decrease value of reward. The output of Reinforcement Programming algorithm is a solution of given problem.

As for optimizing K-means, Reinforcement Programming will randomly positioning a centroid set in first learning. Then, RP will continously optimizing its state by changing centroid point using experiment that represented by reward value. The higher reward value, the higher possibility $\mathrm{RP}$ will take the current point to change. RP also will considering exploration rate to take an action. The greater number of exploration rate set, the higher possibility area will be explored by RP agent. The exection steps of Reinforcement Programming [13] to optimize the initial centroids for Kmeans clustering can be seen as follows:

Let $A=\left\{a_{i} \mid i=1, \ldots, f\right\}$ be attributes of $f$-dimensional vectors and $X=\left\{X_{i} \mid\right.$ $i=1, \ldots, N\}$ be each data of $A$. The K-means clustering separates $X$ into $k$ partitions called clusters $M=\left\{M_{i} \mid i=1, \ldots, k\right\}$ where $M$ I $X$ is $M i=\left\{m_{i j} \mid j=1, \ldots, n\left(s_{i}\right)\right\}$ as members of $s_{i}$, where $n(s i)$ is number of members for si. Each cluster has cluster center of $C=\left\{c_{i} \mid i=1, \ldots, k\right\}$, solution of RP are represented by State $S=\left\{S_{i}\right.$ $\mid i=1, \ldots, k\}$. The folowing execution steps of the proposed algotihm can be described as follows:

1. $\mathrm{S} \leftarrow$ Initiate its algorithm by generating random starting points of initial centroids $C$ and describe as State $S$.

2. Calculate variance value of current State

3. $\mathrm{R} \leftarrow$ random a number beetwen 0 and 1 .

4. If $\mathrm{R}>$ exploration rate, index of State will randomly choosen

5. If $\mathrm{R}<$ exploration rate, index choosen will consider reward value

6. Update and calculate variance value of new $S \leftarrow$ by equitation 2

7. If newsv $<$ sv; $S \leftarrow$ new $S$

8. Calculate reward $\leftarrow$ by equitation 3

9. If newsv $>\mathrm{sv}$

10. Calculate reward with giving a punishment $\leftarrow$ see equitation 4 Change direction

11. If $\mathrm{i}<$ iteration, back to step 3 .

After processing, it will generate the designated initial centroid $\mathrm{Cp}$ where $\mathrm{p}=1,2, \ldots, \mathrm{k}$. Then, we can apply it as initial centroids for K-means clustering. The experiment result will perform the accuracy of proposed method.

\subsection{Multiband image clustering using K-means}

In this section, the clean data from data-preprocessing function will be reads and automatically splits into clusters from the images by color information. 


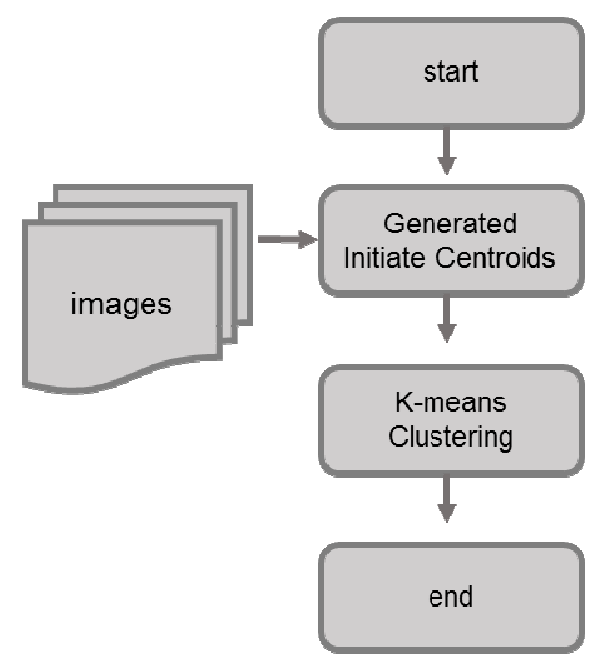

Figure 3. Processing flow of multispectral image Clustering

Figure 3 shows flow of multispectral image clustering. First, this function segments pixels of a satellite images into groups. In this coloredarea, the pixels with close distance in space and color are joined. Then, the pixels divided into specified number of groups by color clustering. This function leads the extraction specific geographical result area from satellite images. As for color clustering, the function divides the pixels of satellite image into specified number of groups by K-means algorithm (Figure 4). Kmeans algorithm works by calculating data and centroid then grouped with pixels which choose the same centroids.

\subsection{Clustered-Image Difference Calculation}

Difference calculation function creates difference-image from the multiband image clustering result. First, from the result of clustering, the function extracts the specified colored-area for extractng difference area. Then, by comparing the extracted area beetwen temporal images, the function will produce an increasing and decreasing area. By drawing each area by specified color, the function will displays difference-images result from calculation process. 


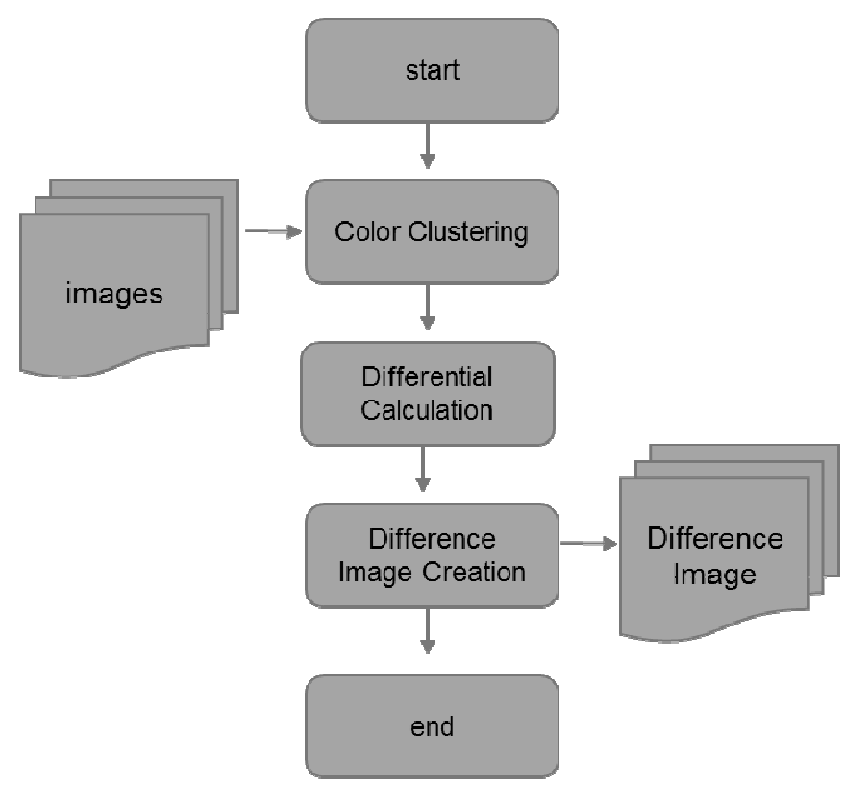

Figure 4. Processing flow of Difference Calculation

\section{EXPERIMENT AND ANALYSIS}

In order to analyse the nature change in deforestration, this experiment collect multiband image in some interval time. The data were taken from glovis.landsat were consist of 7 bands. We apply normal K-mean and K-means using Reinforcement Programming for segementing this multiband satellite images. The following experiment also analyse accuracy and ability of the proposed method for clustering and calculating the land change in several time. The imagery data used in this experiment are multispectral images from Landsat imaginary, and as for the study area used in this paper is Medawai, Borneo (Figure 5) from 2008 until 2012.

Medawai is an area in Borneo that has noticable deforestration activity in last 10 years. Applicating Kmeans for clustering forest and land area in Medawai will give efficient effort for measuring deforestration area. In Medawai, there are three clusters indicating difference nature creature which consist of vegetation, water, and bare soil.

Using K-means, the result will separate multiband satellite image two classes over sources data that will be clustered. The important element for analysist is vegetation area, it used for calculation function to measure forest that exist on recent year. Fitness value of cluster calculated by variance value of a cluster. Smallest number of cluster variance will show the best cluster performance. 


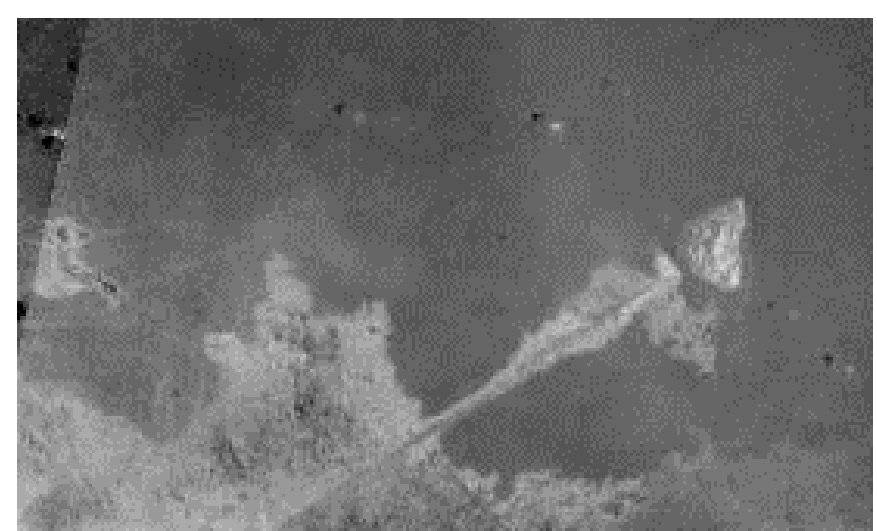

Figure 5. An experiment area: Medawai, Borneo, Indonesia.

This paper shows the optimum result from solving K-means using Reinforcement Programming for optimization. Experiment has been done 100 times, then clustering performance of the proposed method is compared with normal K-means.

\subsection{Study area}

The data sets used for comparison experiments consist of 6 bands. The experiments are performed over 2D maps dataset. This dataset will be proceed in K-means to produce a clustered area. The data set samples are to be tested using K-Means using Reinforcement Programming and normal Kmeans. The use of Reinforcement programming will accelerate accuracy value of K-means performance. In this experimental, this six bands were used as one dataset. This six dataset are composed from band 1, band 2, band 3, band 4 , band 5 , and band 7 . Band 6 could not be implied as resource data, it is because band 6 showing unclear captured data. So, if we include band 6 to system,

In Figure 7 and Figure 8 performs the results between our proposed methods with normal K-means which using random initialization. It is found that the clustering result from our proposed method can make better separated cluster than the random initialization K-means. 


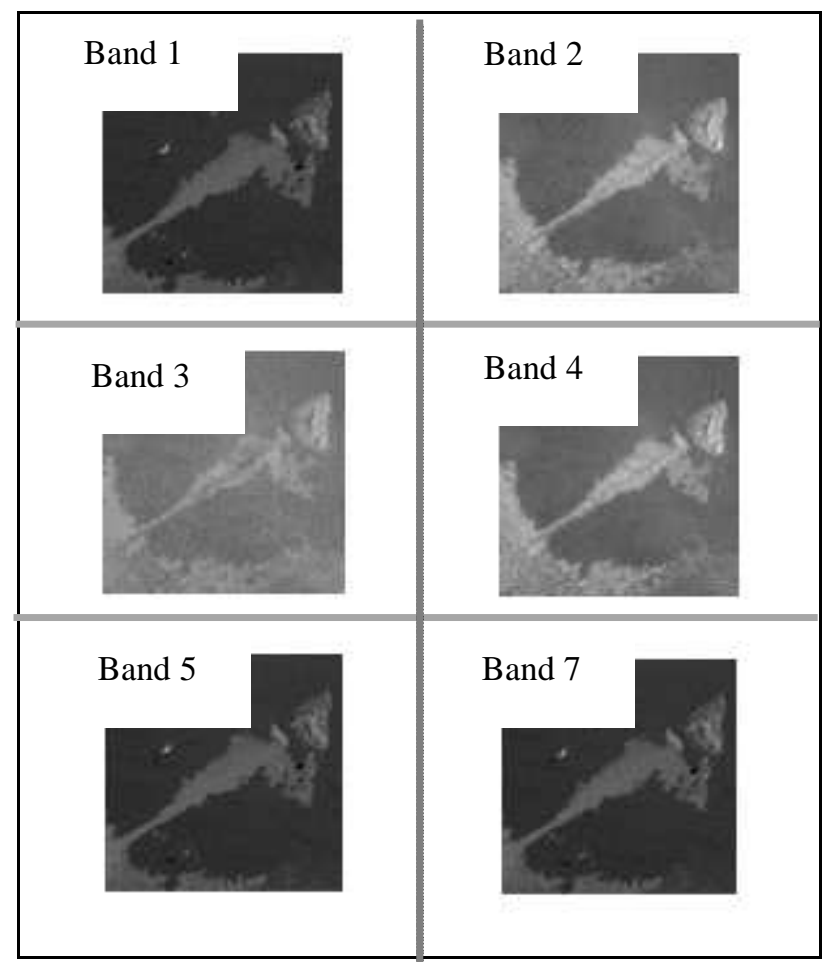

Figure 6. Six Band of Medawai, Borneo

\subsubsection{Experimental Comparison}

The following variance factors (initiate by $\mathrm{V}$ ) is defined as a performance measurement in this experiments. Variance constraint [14] can express the density of the clusters with variance within cluster and variance between clusters [15]. The ideal cluster has minimum variance within clusters $\left(\mathrm{V}_{\mathrm{w}}\right)$ to express internal homogeneity and maximum variance between clusters $\left(\mathrm{V}_{\mathrm{b}}\right)$ to express external homogeneity [16].

The average error and average time taken by the K-means from two different algorithms will be proceed and compared, it is used to find the best algorithm for solving multispectral images. The two algorithm that will be compared are K-means using Reinforcement Programming and normal Kmeans. Table 1 and Table 2 will show the result and performance of each algorithm.

As for the experiment result, we ran total 100 times experiment. It is shows in Table 1 and 2 . We ran 10 times for the 10 iteration experiment and produced weak stability, 10 times for 50,100 and 150 iteration and produced high stability for solving problem.

\section{Reinforcement Programming}

Figure 7 shows that Reinforcement Programming increase the accuracy and has more stability after reach greater number of iteration. It is because reinforcement programming is gaining its experience in beginning of iteration, and after reach some number of iteration Reinforcement programming became clever and can reach better solution by its own 
knowledge And Reinforcement Programming shows good performance to solve minimization problem.

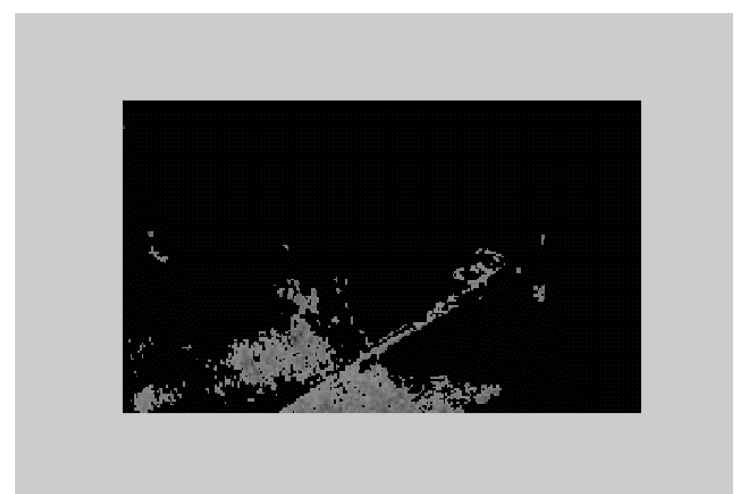

Figure 7. Bare soil distribution using K-means with RP

\section{Normal K-means}

The purple colour of Figure 8 shows soil distribution in Medawai, Borneo, Indonesia. The experiment has been done in 10 times nd shows the high number of accuracy by using variance as the clustering measurement. In other hand, this experiment also compared to another algorithm for clustering, the others are clustering without optimization. It will use random centroid value. The result of this experiment can be seen in Figure 8.

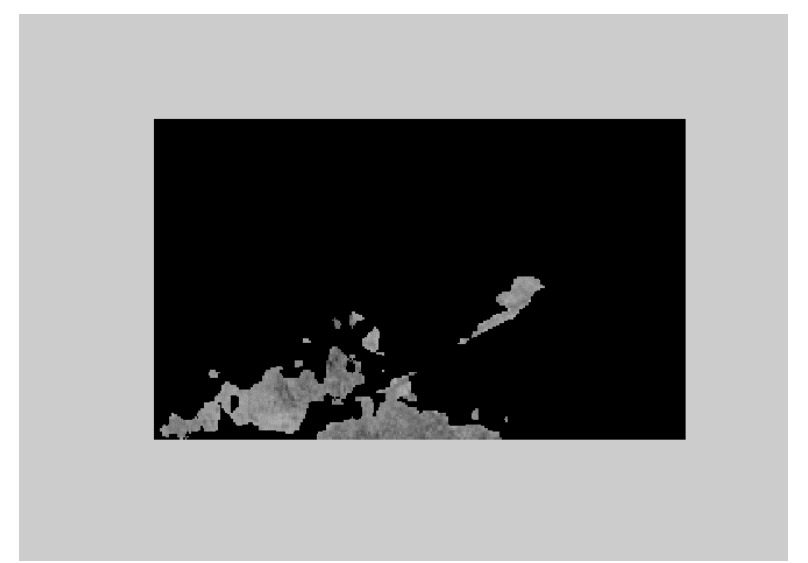

Figure 8. Bare soil distribution using normal K-means

\section{Comparison between RP and normal K-means}

The purple colour of Figure 7 and Figure 8 shows soil distribution in Medawai, Borneo, Indonesia. The accurate result of soil clustering can be shown using K-means with Reinforcement Programming as optimization of K-means, in first initiated centroid. Figure 9 shows complete reshaping images using k-means with RP as optimization centroid. The accurate soil area also shown in solid colour to highlighting the soil area that will be main point for this clustering methods result. 
It is representing that K-means using Reinforcement Programming has a good performing rather than normal k-means.

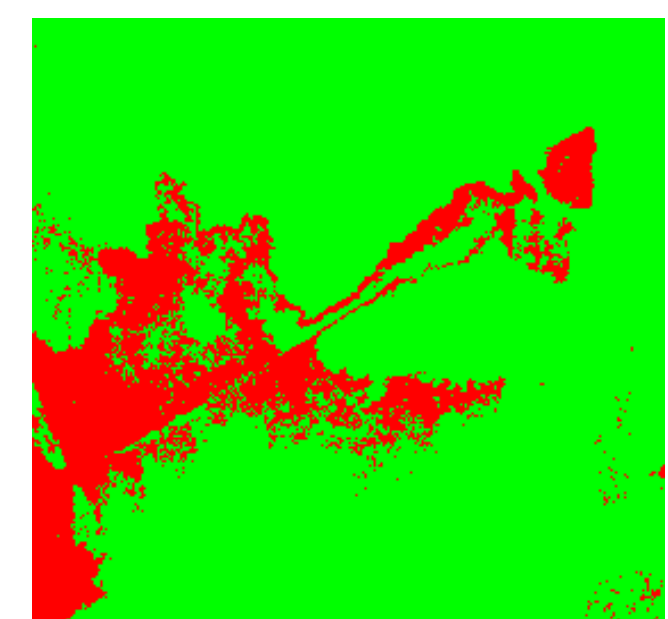

Figure 9. Reshaping image after clustered by K-means with RP

In other hand, we also compared the result with normal K-means. The result of normal K-means in Figure 10 shows value of normal K-means was not as good as Reinforcement Programming optimization

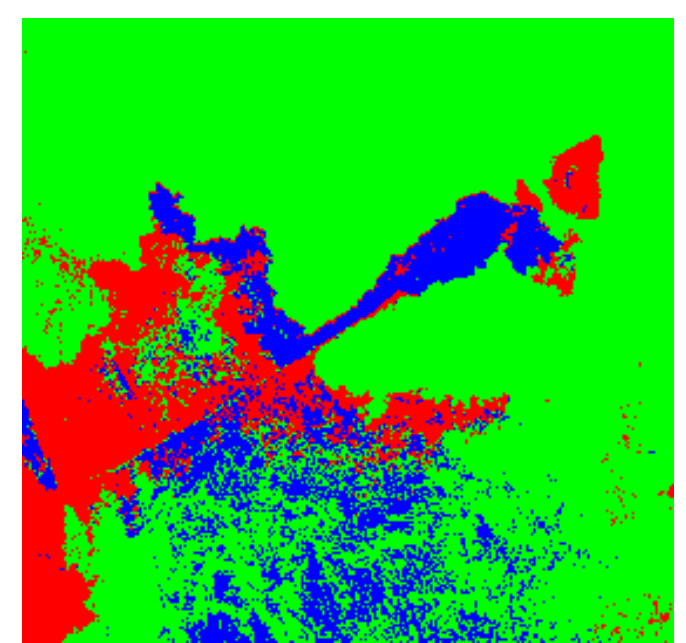

Figure 10. Reshaping image after clustered by normal K-means

As for experiment, we did 10 times of experiment for clustering multispectral images using RP and shown in Table 1, it is also repesent a value of overall cluster variance in each experiment. 
Table 1. Experimental Result of K-means using

Reinforcement Programming with overall cluster variance

\begin{tabular}{|c|c|c|}
\hline Experiment 1 & Experiment 2 & Experiment 3 \\
\hline & & \\
\hline & & \\
\hline & & \\
\hline & & \\
\hline 0.023202607862 & 0.023202607862 & 0.023202607862 \\
\hline Experiment 4 & Experiment 5 & Experiment 6 \\
\hline & Experiment 8 & Experiment 9 \\
\hline & & \\
\hline 0.023202607862 & 0.04160416667 & \\
\hline
\end{tabular}

As for result of experiment using normal K-means could be identify in Table 2 , where contains variance calculation in every experiment.

Table 2. Experimental Result of normal K-means cluster variance

\begin{tabular}{|c|c|c|}
\hline Experiment 1 & Experiment 2 & Experiment 3 \\
\hline 0.08160416667 & 0.023202607862 & 0.023202607862 \\
\hline Experiment 4 & Experiment 5 & Experiment 6 \\
\hline 0.06160416667 & 0.06160416667 & 0.023202607862 \\
\hline Experiment 7 & Experiment 8 & Experiment 9 \\
\hline 0.02462607862 & 0.04350416667 & 0.04350416667 \\
\hline
\end{tabular}

The acuration of clustering result in calculating deforestration area can be shown from experiment of Reinforcement Programming as optimization of K-means, in first initiated centroid. For validation of clustering result of each experiment variance of all cluster has been calculated and show in Table 3 . 
Table 3. Performance of normal K-Means and K-means using Reinforcement Programming

\begin{tabular}{|c|c|c|}
\hline Parameter & Normal K-means & K-means using RP \\
\hline $\begin{array}{c}\text { Execution Time (min) } \\
\text { Average } \\
\text { Variance }\end{array}$ & 14 & 11 \\
\hline Average Iteration for fixed centroid & $4.44906082 \times 10^{2}$ & $2.42026078 \times 10^{2}$ \\
\hline
\end{tabular}

As for result of comparison process, Reinforcement Programming shows a good performance to optimizing K-means. Normal K-means shows longer computational time compared to K-means which optimized by Reinforcement Programming.

\subsubsection{Differential Calculation}

Figure 10 shows the diference extraction results after clustering processing using Reinforcement Programming. The number of cluster in study area is 2 . The focused used colors are green, menas as forest area. And Orange colour as retreated area. The results show that the forest area, indicated in orange colour show a minor degradation number of forest from 2000 until 2005. At 2006 the number of deforestration dramatically increased and left a big damage area. As for 2007 until 2008, deforestration still shows in medawai with a big difference between 2006 and 2007. At this time range, the differences could indicating the major deforestration activity has been done between 2006 until 2009. And the number of deforestration area keep growing in late of 2009 until 2010. At 2010 until 2013, the study area shows there is no change in forest area. Whether for reforestration or deforestration. After 2014 the area in context of deforestration has gradually decreased and at this point reforestration seems to be successfully happen, though the deeper analysis with forest specialist is needed to ensure the real area of reforestration. We need to examine the more details and check with more image forward to ensure reforestration activity in study area by years. The Figure 10 shows the number of deforestration area in medawai Borneo from 2001 until 2013. As clustering result from Figure 10, the deforestration has gradually increase start from 2006 until 2011. But, it is restored to reforestration activity in 2012 . Where green space indicating forest land and orange space indicating damage area or deforestration area. 


\begin{tabular}{|c|c|c|}
\hline 2001 & 2002 & 2003 \\
\hline & & \\
\hline $8.113 \mathrm{~km}^{2}$ & $40.520 \mathrm{~km}^{2}$ & $39.177 \mathrm{~km}^{2}$ \\
\hline 2004 & 2006 & 2007 \\
\hline & & \\
\hline & & \\
\hline $105.43 \mathrm{~km}^{2}$ & $106.39 \mathrm{~km}^{2}$ & 2010 \\
\hline 2008 & 2009 & \\
\hline & & \\
\hline $106.39 \mathrm{~km}^{2}$ & & $106.39 \mathrm{~km}^{2}$ \\
\hline 2011 & $165.44 \mathrm{~km}^{2}$ & 2013 \\
\hline & 2012 & \\
\hline & & $101.4869 \mathrm{~km}^{2}$ \\
\hline
\end{tabular}

Figure 10. The final result of differential extraction and difference-image creation (with 2 cluster. Where green shows the forest area and Orange shows the retreated forest area by years)

The area calculated by counting pixel in orange space and multiply by image scale where landsat satellite image was taken. The graphics in Figure 11, shows the trend of deforestration in medawai, Borneo in past 10 years by using Kmeans with reinforcement Programming.

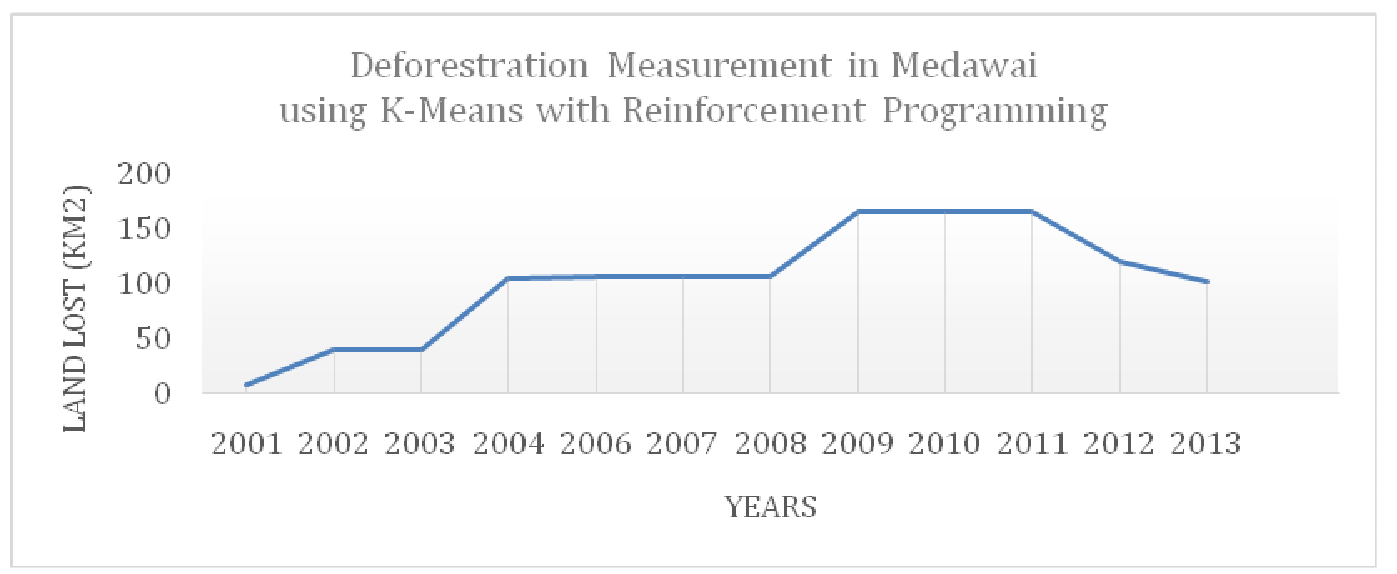

Figure 11. Graph of Deforestration Measurement in Medawai Using K-Means with Reinforcement Programming 
From Figure 11 we could identify deforestration area in past 10 years, the most significant nature change of deforestration happen in 2004 and 2009. In this years, deforestration could be happen because of the ilegal logging or another human activity, though we still need the expert data to support this hypothesis. In other hand the deforestration activity in 2010 start to reach stagnant number. As from graph in 2012 deforestration area slowly decreased and present smaller area of deforestration, it could be reforestration activity from local goverment or the growth of youth tree through rain season.

\section{CONCLUSION}

Multispectral image delivers great source of data for studying temporal changeability of earth environment. But, Multispectral images consist of big data, where difficult to segments because the large size of data. In other hand it is widely reported that K-means clustering algorithm is such a promising clustering methods because of the ability to handle big data, yet suffers from initial centroids. The main purpose is to optimize the designation of the initial centroids for K-means clustering so K-means could run better and give improvement in solving accuracy problem. Therefore this paper proposed Reinforcement Programming as K-means Optimization. This algorithm is based on Reinforcement Learning, which giving solution considering experiment and exploitation of its study area.

Reinforcement Programming has capability of convergence and behaviour to solve k-means problem by using exploration and exploitation. Finally, when comparing the experimental result as for Clustering Result, Kmeans using Reinforcement Programming shows a good performance compared to normal K-means. It is shows that K-means with Reinforcement Programming accuracy is higher than normal k-means. In 10 times experimental trial, we calculate that K-means using Reinforcement Programming present a stable good performance while k-means could fall in bad performance once or twice. The common hypothesis of good accuracy algorithm is always time consumming. Therefore In this paper the computational time has neatly observe. As for experimental result K-means with Reinforcement Programming could present a small difference amount of execution time between K-means with Reinforcement Programming and Normal K-means. And look at the experiment result we could state that Kmeans with Reinforcement Programming successfully optimizing K-means for clustering multispectral Images.

This paper also presented differential computing methods for satellite images and K-means optimization using Reinforcement Programming. As the comparisson result between normal K-means and K-means using Reinforcement Programming shows the better performance of clustered image after using Reinforcement Programming. We examined the feasibility, applicability and effectiveness for environmental change detection and visualization with several experiments. Experiment result for Reinforcement 
Programming as K-means optimization perform the accuracy and improved clustering result as compared to normal K-means.

\section{REFERENCES}

[1] James A. Shine and Daniel B. Carr, A Comparison of Classification Methods for Large Imagery Data Sets, JSM 2002 Statistics in an ERA of Technological Change-Statistical computing section, New York City, pp.3205-3207, 11-15 August 2002.

[2] Bram Bakker \& Jurgen Schmidhuber, Hierarchical Reinforcement Learning Based On Subgoal Discovery and Subpolicy Specialization. Technical report, IDSIA, 2003.

[3] Ming tan, Multi-Agent Reinforcement Learning: independent vs cooperative agent, GTE Laboratories Incorporated.

[4] C.Immaculate Mary, dr. S.V. Kasmir Raja, REFINEMENT OF CLUSTERS FROM K-MEANS WITH ANT COLONY OPTIMIZATION, Journal of Theoretical and Applied Information Technology, 2005-2009.

[5] Abhijit Gosavi, A Reinforcement Learning Algorithm Based On Policy Iteration For Average Reward: Empirical Results With Yield Management And Convergence Analysis, Kluwer Academic Publishers, 2004.

[6] Leslie Pack Kaelbling, Michael L. Littman, Andrew W. Moore. Reinforcement Learning: A Survey, Journal of Articial Intelligence, Artificial Research, 2004

[7] D. Lu, Q. Weng, A survey of image classification methods and techniques for improving classification performance, International Journal of Remote Sensing, Vol. 28, No. 5, pp. 823-870, January 2007.

[8] M. Govender, K. Chetty, V. Naiken and H. Bulcock, A comparison of satellite hyperspectral and multispectral remote sensing imagery for improved classification and mapping of vegetation, Water $S A$, Vol. 34, No. 2, April 2008.

[9] Jasinski, M. F., Estimation of subpixel vegetation density of natural regions using satellite multispectral imagery, IEEE Transactions on Geoscience and Remote Sensing, Vol. 34, pp. 804-813, 1996.

[10] James Theiler and Galen Gisler., A contiquity-enhanced k-means clustering algorithm for unsupervised multispectral image segmentation, Proc SPIE, 1997.

[11] Kohei Arai, Ali Ridho Barakbah., Hierarchical K-means: an algorithm for centroids initialization for K-means, Reports of the Faculty of Science and Engineering, Saga University, Vol. 36, No.1, 2007.

[12] Giri Tejaswi, Manual on Deforestation, Degradation, and Fragmentation using Remote Sensing and GIS, Forestry Department Food and Agriculture Organization of the United Nations, pp. 27-29, 2007. 
[13] Irene Erlyn Wina Rachmawan, Ali Ridho Barakbah, Ira Prasetyaningrum, Yuliana Setiowati, Reinforcement Programming: a function based Reinforcement Learning, The Third IndonesianJapanese Conference on Knowledge Creation and Intelligent Computing (KCIC) 2014, Malang, Indonesia, March 25-26, 2014.

[14] C.J. Veenman, M.J.T. Reinders, E. Backer, A Maximum Variance Cluster Algorithm, IEEE Transactions on Pattern Analysis and Machine Intelligence, Vol. 24, No. 9, pp. 1273-1280, 2002.

[15] S. Ray, R.H. Turi, Determination of Number of Clusters in K-Means Clustering and Application in Colthe Image Segmentation, Proc. 4th ICAPRDT, pp.137-143, 1999.

[16] Ali Ridho Barakbah, Kohei Arai, Identifying Moving Variance to make Automatic Clustering for normal dataset, Proc. IECI Japan Workshop 2004 (IJW 2004), Musashi Institute of Technology, Tokyo, 2004. 